\title{
TRATAMENTO LAPAROSCÓPICO DOS CATETERES DE DIÁLISE PERITONEAL OBSTRUÍDOS
}

\section{LAPAROSCOPIC TREATMENT OF CATHETERS WITH OBSTRUCTION IN PERITONEAL DIALISIS}

\author{
Orlando Ribeiro Prado Filho, TCBC-PR 1 \\ José Miguel Viscarra Obregon ${ }^{2}$ \\ Sergio Seiji Yamada ${ }^{3}$
}

\section{INTRODUÇÃO}

A diálise peritoneal é um método efetivo no tratamento de pacientes com insuficiência renal crônica, porém ainda esta associado a um número significativo de complicações. ${ }^{1}$ A dificuldade de saída do líquido infundido ocorre em cerca de $60 \%$ dos casos, sendo necessária a retirada do cateter em $39 \%$ dos pacientes. $^{2}$

Esta série apresenta a casuística do Serviço de $\mathrm{Ne}$ frologia da Santa Casa de Maringá de pacientes em regime de diálise peritoneal ambulatorial contínua (CAPD), que apresentaram complicações mecânicas no cateter e foram submetidos a operações videoassistidas para correção do problema.

\section{RELATO DO CASO}

Entre junho de 1996 e outubro de 1998 sete procedimentos laparoscópicos foram realizados em seis pacientes listados na Tabela 1.

Tabela 1

Características dos pacientes

\begin{tabular}{c|c|c|l}
\hline Paciente & Idade & Sexo & Causa da insuficiência renal \\
\hline 1 & 52 & M & Rins policísticos \\
2 & 48 & M & Rins policísticos \\
3 & 72 & M & Diabetes mellitus \\
4 & 17 & F & Síndrome hemolítico-urêmica \\
5 & 60 & F & Diabetes mellitus \\
6 & 28 & F & Glomerulonefrite crônica \\
\hline
\end{tabular}

Todos os procedimentos foram realizados com o paciente sob anestesia geral. Após a visibilização do cateter, a causa da obstrução foi avaliada. Após a liberação das aderências no cateter peritoneal sua extremidade distal foi colocada na pelve.

Todos os cateteres foram testados para infusão e saída de solução fisiológica antes da retirada do laparoscópio. A diálise peritoneal foi iniciada no primeiro dia de pós-operatório com um volume de infusão inicial máximo de $1.000 \mathrm{ml}$.

\section{RESULTADOS}

Foram realizados sete procedimentos laparoscópicos em seis pacientes (Tabela 1). Todos os procedimentos foram indicados devido à dificuldade de saída do líquido infundido. Em três pacientes o mesmo cateter foi mantido em diálise peritoneal por tempo superior a 12 meses (Tabela 2).

No paciente número três durante a lise de aderências houve uma pequena laceração no intestino delgado (ID), que foi reparada por procedimento à céu aberto. Foi realizada a sutura da lesão, irrigação copiosa da cavidade abdominal e lise de aderências. Não houve seqüela da enterotomia tendo sido mantido o mesmo cateter para diálise peritoneal.

Na paciente número quatro ocorreu saída de líquido sanguinolento durante a drenagem do líquido infundido, fato que se repetiu por vários dias, sem que houvesse repercussão hemodinâmica, porém com alteração hematimétrica. A paciente foi levada à novo ato operatório video-assistido, tendo sido constatada a presença de um pequeno vaso ainda com sangramento ativo no orifício na parede abdominal, que fora usado para colocação da ótica. Foi realizada a hemostasia por eletrocautério, tendo a paciente evoluído satisfatoriamente no pós-opretório.

1. Professor Assistente da Área de Clínica Cirúrgica do Departamento de Medicina da UEM - PR

2. Professor Auxiliar da Área de Clínica Médica do Departamento de Medicina da UEM e Médico do Serviço de Nefrologia da Santa Casa de Maringá - PR

3. Professor Adjunto da Área de Clínica Médica do Departamento de Medicina da UEM e Médico do Serviço de Nefrologia da Santa Casa de Maringá - PR

Recebido em 24/6/99

Aceito para publicação em 1/2/2000

Trabalho realizado na Área de Clínica Cirúrgica do Departamento de Medicina da Universidade Estadual de Maringá (UEM) e no Serviço de Nefrologia da Santa Casa de Maringá - PR. 
Tabela 2

Operação realizada e intercorrências

\begin{tabular}{ll|ll}
\hline Achados & Procedimento & Resultados & Complicações \\
\hline 1. Aderências & Lise aderências & Funcionante > 20 meses & Nenhuma \\
2. Grande omento & Liberação & Funcionante > 12 meses & Nenhuma \\
3. Intestino delgado & Liberação & Funcionante 3 meses & Laceração I.D. \\
4. Grande omento & Liberação & Reoperação & Hemorragia \\
5. Grande omento & Liberação & Retirado por infecção & Infecção \\
6. Grande omento & Liberação & Funcionante 3 meses & Infecção \\
7. Hemoperitônio & Hemostasia & Funcionante $>20$ meses & Nenhuma \\
\hline
\end{tabular}

\section{DISCUSSÃO}

A diálise peritoneal continua a ganhar adeptos no tratamento dos pacientes em estágio final de insuficiência renal crônica. Apesar de amplamente aceito, o sucesso do procedimento é limitado pelo funcionamento do cateter. ${ }^{2}$

O sucesso da manipulação do cateter com mau funcionamento por laparoscopia é bem documentado em outros trabalhos. ${ }^{3,4,5}$ Esta via permite o exame direto da cavidade abdominal o que facilita que se faça a acurada identificação da causa do mau funcionamento e imediatamente se restaure sua função com retorno rápido às sessões de diálise peritoneal. ${ }^{3}$

Nos pacientes com obstrução à drenagem de líquido o achado usual foi o grande omento ao redor do cateter. ${ }^{2}$ $\mathrm{Na}$ série apresentada a maioria dos casos $(75 \%)$ de falha na drenagem de líquido intra-abdominal deveu-se à obliteração pelo grande omento. Em nenhum caso foi necessário a feitura de omentectomia parcial.

A obliteração do espaço peritoneal por aderências indica a necessidade de procedimento laparoscópico, porém a presença de dor durante a infusão de líquido e an- tecedentes de peritonite diminuem a probabilidade de sucesso de correção pelo procedimento. ${ }^{3}$

A morbidade alcançou a taxa de $28,3 \%$ com duas complicações técnicas (laceração entérica e hemorragia) em sete procedimentos. A laceração entérica ocorreu em um paciente que apresentou quadros repetidos prévios de peritonite com várias retiradas e implantes de cateteres. O caso de hemorragia ocorreu no orifício de entrada do trocarte de $10 \mathrm{~mm}$ que foi utilizado para a inserção da ótica, em uma paciente com hipertensão arterial sistêmica grave; foi resolvido com uma nova operação videoassistida onde foi realizada cauterização do vaso.

Concluindo, a operação videoassistida é um útil instrumento no arsenal cirúrgico para o tratamento de pacientes com insuficiência renal e regime de CAPD que necessitam de correção da falha na drenagem do líquido de diálise. O procedimento permite o rápido retorno das sessões de diálise, porém, não é isento de complicações. Com o apuro técnico dos cirurgiões laparoscopistas o sucesso do procedimento beneficiará mais pacientes e permitirá a preservação dos cateteres por longo tempo.

\begin{abstract}
The success of peritoneal dialysis in the treatment of patients with chronic renal failure depends on a proper performance of peritoneal catheter. This study shows the experience from the Service of Surgery from Departament of Medicine of State University of Maringá and the Service of Nephrology from Maria Auxiliadora Hospital, Maringá, in the laparoscopic approach of catheters with obstruction.
\end{abstract}

Key Words: CAPD; Laparoscopy; Peritoneal dialysis; Renal failure.

\section{REFERÊNCIAS}

1. Francis DM, Donnely PK, Veitch PS, et al - Surgical aspects of continuous ambulatory peritoneal dialysis -3 year experience . Br J Surg 1984; 71:225-9.

2. Kimmelstier FM, Miller RE, Molinelli BM, et al - Laparoscopic manangement of peritoneal dialysis catheters. Surg Gynecol Obstet 1993; 176:565-70.

3. Crabtree JH, Fishman A - Laparoscopic epiplopexy of the greater omentum and epiploic appendices in the salvaging on dysfunctional peritoneal dialysis catheters. Surg Laparosc Endosc 1996; 6:176-80.
4. Davis R, Young J, Diamond D, et al - Manangement of chronic peritoneal catheter malfunction. Am J Nephrol 1982; 2:85-90.

5. Leung LC, Yui MK, Man CW, et al - Laparoscopic manangement of Tenckhoff catheters in continuous ambulatory peritoneal dialysis. A one-port technique. Surg Endosc 1998; 12:891-3.

\section{ENDEREÇO PARA CORRESPONDÊNCIA}

Dr. Orlando Ribeiro Prado Filho

Rua Silva Jardim, 560 - Centro

87013-010 - Maringá-PR 